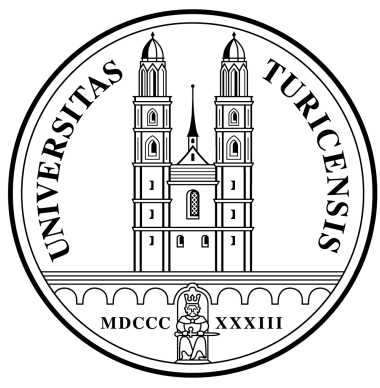

Institute for Empirical Research in Economics

University of Zurich

Working Paper Series

ISSN 1424-0459

Working Paper No. 83

\title{
Market Selection of Financial Trading Strategies: Global Stability
}

Igor V. Evstigneev, Thorsten Hens and Klaus Reiner Schenk-Hoppé

July 2001 


\title{
Market Selection of Financial Trading Strategies: Global Stability*
}

\author{
Igor V. Evstigneev ${ }^{\dagger}$ \\ Thorsten Hens ${ }^{\ddagger}$ \\ Klaus Reiner Schenk-Hoppé ${ }^{\S}$
}

July 11, 2001

\begin{abstract}
In this paper we analyze the long-run dynamics of the market selection process among simple trading strategies in an incomplete asset market with endogenous prices. We identify a unique surviving financial trading strategy. Investors following this strategy asymptotically gather total market wealth. This result generalizes findings by Blume and Easley (1992) to any complete or incomplete asset market.
\end{abstract}

\section{JEL-Classification: D52, D81, D83, G11}

Keywords: portfolio theory, evolutionary finance, incomplete markets

\footnotetext{
${ }^{*}$ Financial support from Swiss Research Foundation for Banking and Finance is gratefully acknowledged.

†igor.evstigneev@man.ac.uk. School of Economic Studies, University of Manchester, Oxford Road, Manchester M13 9PL, UK.

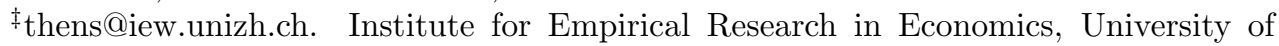
Zurich, Blümlisalpstrasse 10, 8006 Zürich, Switzerland.

§klaus@iew.unizh.ch. Institute for Empirical Research in Economics, University of Zurich, Blümlisalpstrasse 10, 8006 Zürich, Switzerland.
} 


\section{Introduction}

In their seminal paper Blume and Easley (1992) studied the market selection mechanism in an asset market model with endogenous prices. Investors follow simple trading strategies (i.e. the proportion of wealth invested in each asset is fixed over time) and reinvest their payoffs in each period. The market equilibrium process enforces a redistribution of market wealth among the traders. Blume and Easley (1992) showed that in a market in which securities have diagonal payoffs (i.e. in each state exactly one asset has a non-zero payoff ${ }^{1}$ ) the unique survivor of the market selection process is "betting your beliefs." That is those investors who divide income proportionally to the probabilities of the states have the highest growth rate of wealth in any population of simple trading strategies and will therefore accumulate the total market wealth in the long run.

With exogenous prices (fixed to one for every asset) this remarkable property of the "betting your beliefs"-rule has been known ever since Breiman (1961). This rule can be generated by maximizing the expected logarithm of relative returns which in turn is known as the Kelly rule, (Kelly 1956).

In this paper we extend the above model to asset markets with incomplete payoff structure. We show that, in any incomplete market without redundant assets, there is a unique surviving financial trading strategy which asymptotically gathers total market wealth. According to this trading strategy, one should divide income proportionally to the expected relative returns of the assets. In the case of diagonal securities, this means "betting one's beliefs."

The results presented in this paper bring the portfolio theory of Blume and Easley (1992) (which is based on market selection) closer to a possible application. Indeed the new strategy is very easy to implement. Assuming ergodicity of the returns a large enough sample of past returns is a good estimator of expected returns and in order to form the best surviving portfolio, nothing more needs to be known about the assets.

Our paper also contributes to the problem of log-optimum investment policies in which trading strategies with maximum expected (exponential) growth rate are searched for. This maximum growth perspective is explored e.g. by Algoet and Cover (1988), Aurell, Baviera, Hammarlid, Serva, and Vulpiani (1998), Browne and Whitt (1996), Cover (1991), Hakansson (1970), Hakansson and Ziemba (1995), Karatzas and Shreve (1998), Thorp (1971),

\footnotetext{
${ }^{1}$ An example are Arrow securities.
} 
among others. In this approach the price process is treated as exogenous, which is common practice in mathematical finance. However, from an equilibrium point of view this assumption is not satisfactory because it ignores any equilibrium consequences. In our model we calculate the market-clearing price in every period based on the existing trading strategies and the associated market wealth of investors. Thus the price-mechanism plays a central role in the market selection process here. In this view our model endogenizes prices in the above-mentioned approach. The possibility of "evolutionary spite," which transmits through prices, no longer favors investors maximizing the expected logarithm of pay-offs when markets are incomplete.

For recent results in the case of endogenous savings and non-simple trading strategies see Blume and Easley (2000) and Sandroni (2000). These papers show that in the case of complete markets among all infinite horizon expected utility maximizers those who happen to have rational expectations will eventually dominate the market. This interesting result holds irrespectively of the investors' risk aversion characteristics. The intuition goes that differences in investors expectations determine their savings rates which are essential for the growth of the investors' wealth.

Finally let us remark that the result of this paper is complementary to that of Hens and Schenk-Hoppé (2001) who show that the trading strategy mentioned above is the unique locally stable trading strategy for general ergodic states of the world.

Section 2 introduces the model, and Section 3 presents the main result of this paper. The proof of the auxiliary results are given in the Appendix.

\section{The Investment Model}

Except for the more general asset structure, we use the same model as in Blume and Easley (1992). Time $t=1,2, \ldots$ is discrete, and uncertainty is modeled by a sequence $s_{1}, s_{2}, \ldots$ of independent identically distributed random variables (states of the world), taking values in a finite space $S$. In every period, the states are drawn according to the probability distribution $p=\left(p_{s}\right)_{s \in S}, p_{s}>0$. We denote by $P$ the product measure of $p$ constructed on the space of sample paths $\left(s_{t}\right)_{t=1,2, \ldots}$. Further $E$ denotes the expectation with respect to the measure $P$.

There are $i=1, \ldots, I$ investors initially endowed with wealth $w_{0}^{i}>0$.

Assets $k=1, \ldots, K$ live for one period only but are identically reborn 
in every period. They yield the non-negative return $A_{k}(s)$ at state $s$, and we assume that $A_{k}(s) \neq 0$ for at least one $s$. Moreover, we assume that $\sum_{k} A_{k}(s)>0$ for all $s$.

At each time $t$, every investor $i$ selects a portfolio

$$
\theta_{t}^{i}\left(s^{t}\right)=\left(\theta_{1, t}^{i}\left(s^{t}\right), \ldots, \theta_{K, t}^{i}\left(s^{t}\right)\right) \in \mathbb{R}_{+}^{K}
$$

based on the observation of the history $s^{t}=\left(s_{1}, \ldots, s_{t}\right)$.

Let $\rho_{k, t}\left(s^{t}\right)$ be the price of asset $k$ in period $t$ given history $s^{t}$. Then we can define the agent's budget shares as

$$
\lambda_{k, t}^{i}\left(s^{t}\right)=\frac{\rho_{k, t}\left(s^{t}\right) \theta_{k, t}^{i}\left(s^{t}\right)}{w_{t}^{i}\left(s^{t}\right)}
$$

where $w_{t}^{i}\left(s^{t}\right)$ is the investor's wealth, i.e.,

$$
w_{t}^{i}\left(s^{t}\right)=\sum_{k=1}^{K} A_{k}\left(s_{t}\right) \theta_{k, t-1}^{i}\left(s^{t-1}\right) .
$$

For each $i$, we call the sequence of budget shares $\left(\lambda_{k, t}^{i}\left(s^{t}\right)\right)_{t=1,2, \ldots}$ a trading strategy of investor $i$. A completely mixed trading strategy assigns a positive percentage of wealth $\lambda_{k, t}^{i}\left(s^{t}\right)$ to every asset $k=1, \ldots, K$ for all $t$ and $s^{t}$ (and so $w_{t}^{i}\left(s^{t}\right)>0$ for all $\left.t, s^{t}\right)$. Following Blume and Easley (1992), we focus on trading strategies that are completely mixed and, moreover, simple: the budget shares $\lambda_{k, t}^{i}\left(s^{t}\right)=\lambda_{k}^{i}>0$ are supposed to be constant, i.e. independent of $t$ and $s^{t}$.

The investor's budget restriction is

$$
\sum_{k=1}^{K} \rho_{k, t}\left(s^{t}\right) \theta_{k, t}^{i}\left(s^{t}\right)=w_{t}^{i}\left(s^{t}\right)
$$

in any period in time $t=0,1,2, \ldots$. Hence every $\lambda^{i}=\left(\lambda_{1}^{i}, \ldots, \lambda_{K}^{i}\right)$ is in the unit simplex $\Delta^{K}=\left\{a \in \mathbb{R}_{+}^{K} \mid \sum_{k} a_{k}=1\right\}$.

By our assumptions on the returns of the assets, the wealth of any completely mixed trading strategy always remains positive. This does not mean, however, that every completely mixed trading strategy will survive, i.e. will have a positive wealth almost surely also in the limit for $t \rightarrow \infty$. It will be proved later on that, among such strategies, there will be a single survivor. Note also that in the definition of next period wealth we have assumed 
that no investor saves or withdraws any money along the process. Our results carry over to the case of saving rates which are constant over time and identical among investors.

Define aggregate wealth in state $s$ at period $t$ by $w_{t}\left(s^{t}\right)=\sum_{i} w_{t}^{i}\left(s^{t}\right)$. Then market shares are given by $r_{t}^{i}\left(s^{t}\right)=w_{t}^{i}\left(s^{t}\right) / w_{t}\left(s^{t}\right)$ and we can define normalized prices by $q_{k, t}\left(s^{t}\right)=\rho_{k, t}\left(s^{t}\right) / w_{t}\left(s^{t}\right)$. The relative payoffs are $R_{t}^{k}\left(s^{t}\right)=A_{k}\left(s_{t}\right) / w_{t}\left(s^{t}\right)$. This allows to write the investor's next period relative wealth equivalently as

$$
r_{t+1}^{i}\left(s^{t+1}\right)=\sum_{k=1}^{K} R_{t+1}^{k}\left(s^{t+1}\right) \frac{\lambda_{k}^{i} r_{t}^{i}\left(s^{t}\right)}{q_{k, t}\left(s^{t}\right)} .
$$

In this model, we assume that the supply of every asset is exogenously given. Without loss of generality, by appropriate choice of the returns, we can safely assume that assets are in fixed supply of one unit each. Hence equilibrium of supply and demand requires that

$$
\sum_{i=1}^{I} \theta_{k, t}^{i}\left(s^{t}\right)=1 \text { for all } t, k, \text { and } s^{t} .
$$

Note that equilibrium implies $w_{t}\left(s^{t}\right)=\sum_{k=1}^{K} A_{k}\left(s_{t}\right)$, and so we can write $w_{t}\left(s^{t}\right)=w\left(s_{t}\right)$, i.e., the aggregate wealth is a function of the current state $s_{t}$ rather than of the whole history $s^{t}$ and, moreover, this function does not explicitly depend on $t$. Similarly, $R_{t}^{k}\left(s^{t}\right)=R_{k}\left(s_{t}\right)$, and, furthermore, $\sum_{k=1}^{K} R_{k}\left(s_{t}\right)=1$. Moreover, the normalized prices are determined by the market shares according to

$$
q_{k, t}\left(s^{t}\right)=\sum_{i=1}^{I} \lambda_{k}^{i} r_{t}^{i}\left(s^{t}\right) .
$$

This finishes the description of the model. In the next section we analyze the evolution of market shares.

\section{A Global Stability Result}

According to the above model, the market selection process is given by the following random dynamical system, describing the evolution of the market 
shares:

$$
r_{t+1}^{i}\left(s^{t+1}\right)=\sum_{k=1}^{K} R_{k}\left(s_{t+1}\right) \frac{\lambda_{k}^{i} r_{t}^{i}\left(s^{t}\right)}{\sum_{i=1}^{I} \lambda_{k}^{i} r_{t}^{i}\left(s^{t}\right)}, i=1,2, \ldots, I .
$$

We say that an investor $i$ (or the strategy $\lambda^{i}$ used by $i$ ) is a single survivor if, for any strictly positive initial vector $r_{0}$, we have $r_{t}^{i} \rightarrow 1$ almost surely (a.s.) and $\lambda_{t}^{j} \rightarrow 0$ a.s. when $j \neq i$. Denote by $e_{i}$ the vector in $\mathbb{R}^{I}$, whose coordinates are equal to 0 except for the $i$ th coordinate which is equal to 1 . If investor $i$ is a single survivor, then all the random paths (1) are attracted to $e_{i}$ a.s., regardless of the initial state. In this sense, $e_{i}$ is a globally stable fixed point of the random dynamical system under consideration. The main result - Theorem 1 below - indicates a strategy $\lambda^{*} \in \Delta^{I}$ such that, under quite general assumptions, the following assertion holds: if investor $i$ uses the strategy $\lambda^{*}$, whereas all the other investors use different strategies, $\lambda^{j} \neq \lambda^{*}$, then $i$ is a single survivor.

Define

$$
\bar{R}_{k}=E R_{k}\left(s_{t}\right)\left[=\sum_{s \in S} p_{s} R_{k}(s)\right] .
$$

Set $\lambda^{*}=\left(\lambda_{k}^{*}\right)$, where

$$
\lambda_{k}^{*}=\bar{R}_{k}, k=1, \ldots, K
$$

and assume that $\bar{R}_{k}>0$ for each $k$. Suppose there are no redundant assets in the market, i.e., the functions $R_{1}(s), \ldots, R_{K}(s)$ of $s \in S$ are linearly independent then we can state the following theorem.

Theorem 1 Let investor $i$ use the strategy $\lambda^{i}=\lambda^{*}$, while all the other investors $j \neq i$ use strategies $\lambda^{j} \neq \lambda^{*}$. Then investor $i$ is the single survivor.

This theorem is valid for any strictly positive vector of initial wealth $r_{0}$, which yields the global stability of the state $e_{i}$, as mentioned above.

Note that the assumption of non-existence of redundant assets is essential. This assumption can be fulfilled only if the number of elements in $S$ is not less than $K$. Thus it cannot hold in the deterministic case - when $S$ consists of a single point. In the latter case, it is easy to construct an example when the investor $i$ using the strategy $\lambda^{*}$ is not a single survivor. Indeed, suppose $i=1$ and the strategy $\lambda^{1}=\lambda^{*}$ is representable in the form $\lambda^{*}=\sum_{j=2}^{I} b_{j} \lambda^{j}$, where $b=\left(b_{j}\right) \in \Delta^{I-1}, b_{j}>0$. Then, for any $\kappa \in(0,1)$, we 
can represent $\lambda^{*}$ as $\sum_{j=1}^{I} a_{j} \lambda^{j}$, where $a_{1}=1-\kappa$ and $a_{j}=\kappa b_{j}, j \geq 2$. In the deterministic case, the values $R_{k}(s)=\lambda_{k}^{*}$ do not depend on $s$, and we can see from (1) that the vector $r=\left(a_{j}\right) \in \Delta^{I}$ is a fixed point of the dynamical system in question for each $\kappa \in(0,1)$. This leads to the failure of (global as well as local) stability of the point $e_{1}$.

Proof of Theorem 1. The proof proceeds in several steps. We also employ an auxiliary result whose proof is relegated to the Appendix.

1 st step. Without loss of generality, we may assume that $i=1$ and hence $\lambda_{k}^{1}=\lambda_{k}^{*}$. Define

$$
q_{k, t}\left(s^{t}\right)=\sum_{m=1}^{I} \lambda_{k}^{m} r_{t}^{m}\left(s^{t}\right), \zeta_{k, t}\left(s^{t}\right)=\frac{\sum_{i=2}^{I} \lambda_{k}^{i} r_{t}^{i}\left(s^{t}\right)}{1-r_{t}^{1}\left(s^{t}\right)}
$$

By using (1), we can write (omitting the arguments $s^{t}$ and $s^{t+1}$ )

$$
\frac{1-r_{t+1}^{1}}{1-r_{t}^{1}}=\frac{\sum_{i=2}^{I} r_{t+1}^{i}}{1-r_{t}^{1}}=\sum_{k=1}^{K} R_{k}\left(s_{t+1}\right) \frac{\left(1-r_{t}^{1}\right)^{-1} \sum_{i=2}^{I} \lambda_{k}^{i} r_{t}^{i}}{q_{k, t}}=\sum_{k=1}^{K} R_{k}\left(s_{t+1}\right) \frac{\zeta_{k, t}}{q_{k, t}}
$$

Observe that

$$
\sum_{k=1}^{K} \zeta_{k, t}=\frac{\sum_{i=2}^{I} \sum_{k=1}^{K} \lambda_{k}^{i} r_{t}^{i}}{1-r_{t}^{1}}=\frac{1-r_{t}^{1}}{1-r_{t}^{1}}=1
$$

and

$$
q_{k, t}=\sum_{m=1}^{I} \lambda_{k}^{m} r_{t}^{m}=\lambda_{k}^{1} r_{t}^{1}+\left(1-r_{t}^{1}\right) \frac{\sum_{i=2}^{I} \lambda_{k}^{i} r_{t}^{i}}{1-r_{t}^{1}}=\lambda_{k}^{1} r_{t}^{1}+\zeta_{k, t}\left(1-r_{t}^{1}\right) .
$$

Consequently,

$$
1-r_{t+1}^{1}=\sum_{k=1}^{K} R_{k}\left(s_{t+1}\right) \frac{\zeta_{k, t}\left(1-r_{t}^{1}\right)}{\lambda_{k}^{1} r_{t}^{1}+\zeta_{k, t}\left(1-r_{t}^{1}\right)}
$$

while

$$
r_{t+1}^{1}=\sum_{k=1}^{K} R_{k}\left(s_{t+1}\right) \frac{\lambda_{k}^{1} r_{t}^{1}}{\lambda_{k}^{1} r_{t}^{1}+\zeta_{k, t}\left(1-r_{t}^{1}\right)}
$$

The above considerations may be regarded as a reduction of the original, I-dimensional, dynamical system to another system, which is, on the one 
hand, only 2-dimensional, but on the other hand, deals with a process of a more general kind (more general, because $\zeta_{k, t}$ depends on $t$ and $s^{t}$ ).

2nd step. For each $t=1,2, \ldots$, consider the random variable

$$
D_{t}=\ln \frac{r_{t}^{1} / r_{t-1}^{1}}{\left(1-r_{t}^{1}\right) /\left(1-r_{t-1}^{1}\right)}
$$

We have

$$
D_{1}+\ldots+D_{T}=\ln \frac{r_{T}^{1}}{1-r_{T}^{1}}-\ln \frac{r_{0}^{1}}{1-r_{0}^{1}}
$$

Therefore, in order to prove that the investor 1 is a single survivor, it is sufficient to show that

$$
\lim _{T \rightarrow \infty} \frac{1}{T}\left(D_{1}+\ldots+D_{T}\right)>0, \quad \text { a.s. }
$$

From (1), we find

$$
H^{-1} \leq \frac{r_{t+1}^{i}}{r_{t}^{i}} \leq H, \text { for all } i=1, \ldots, I .
$$

where $H=\left(\max _{i, k} \lambda_{i}^{k}\right)\left(\min _{i, k} \lambda_{k}^{i}\right)^{-1}$. This implies

$$
H^{-1} \leq \frac{1-r_{t+1}^{1}}{1-r_{t}^{1}} \leq H
$$

because $1-r_{t}^{1}=\sum_{m=2}^{I} r_{t}^{m}$. Consequently, the random variables $D_{t}$ are uniformly bounded.

We have the following identity

$$
\frac{1}{T} \sum_{t=0}^{T-1} D_{t+1}=\frac{1}{2 T}\left(D_{1}+D_{T}\right)+\frac{1}{2 T} \sum_{t=1}^{T-1}\left(D_{t}+D_{t+1}\right) .
$$

Since the random variables $D_{T}$ are uniformly bounded, it suffices to show that

$$
\lim _{T \rightarrow \infty} \frac{1}{T} \sum_{t=1}^{T} G_{t}>0, \quad \text { a.s. }
$$

where $G_{t}=D_{t}+D_{t+1}$. For each $t \geq 1$, denote by $E\left(\cdot \mid s^{t}\right)$ the operator of conditional expectation given $s^{t}$. Let us write, formally, $E\left(\cdot \mid s^{0}\right)$ for the unconditional expectation $E(\cdot)$. Then we have

$$
\frac{1}{T} \sum_{t=1}^{T} G_{t}=\frac{1}{T} \sum_{t=1}^{T} E\left(G_{t} \mid s^{t-1}\right)+\frac{1}{T} \sum_{t=1}^{T}\left[G_{t}-E\left(G_{t} \mid s^{t-1}\right)\right] .
$$


In view of the uniform boundedness of $G_{t}$, we can apply to the process of martingale differences $B_{t}:=G_{t}-E\left(G_{t} \mid s^{t-1}\right)$ the strong law of large numbers, see e.g. Hall and Heyde (1980, Theorem 2.19), which yields $T^{-1}\left(B_{1}+\ldots\right.$ $\left.+B_{T}\right) \rightarrow 0$ a.s. The proof will be completed if we show that, for some strictly positive constant $\delta$,

$$
E\left[G_{t} \mid s^{t-1}\right] \geq \delta, \text { a.s. for all } t=1,2, \ldots
$$

3rd step. We have

$$
\begin{aligned}
E\left[G_{t} \mid s^{t-1}\right] & =E\left[\left(D_{t+1}+D_{t}\right) \mid s^{t-1}\right] \\
& =E\left[\ln \frac{r_{t+1}^{1} / r_{t}^{1}}{\left(1-r_{t+1}^{1}\right) /\left(1-r_{t}^{1}\right)} \mid s^{t-1}\right]+E\left[\ln \frac{r_{t}^{1} / r_{t-1}^{1}}{\left(1-r_{t}^{1}\right) /\left(1-r_{t-1}^{1}\right)} \mid s^{t-1}\right]
\end{aligned}
$$

By using (2), (3) and the assumption of independence of the random variables $s_{t}$, we find

$$
\begin{aligned}
E\left[G_{t} \mid s^{t-1}\right]= & \sum_{\sigma, s} p_{\sigma} p_{s} \ln \frac{\sum_{k} R_{k}(\sigma) \frac{\lambda_{k}^{1}}{\lambda_{k}^{1} r_{t}^{1}(s)+\zeta_{k, t}(s)\left(1-r_{t}^{1}(s)\right)}}{\sum_{k} R_{k}(\sigma) \frac{\zeta_{k, t}(s)}{\lambda_{k}^{1} r_{t}^{1}(s)+\zeta_{k, t}(s)\left(1-r_{t}^{1}(s)\right)}} \\
& +\sum_{s} p_{s} \ln \frac{\sum_{k} R_{k}(s) \frac{\lambda_{k}^{1}}{\lambda_{k}^{1} r_{t-1}^{1}+\zeta_{k, t-1}\left(1-r_{t-1}^{1}\right)}}{\sum_{k} R_{k}(s) \frac{\zeta_{k, t-1}}{\lambda_{k}^{1} r_{t-1}^{1}+\zeta_{k, t-1}\left(1-r_{t-1}^{1}\right)}}
\end{aligned}
$$

where

$$
\begin{aligned}
\zeta_{k, t}(s) & =\frac{\sum_{i=2}^{I} \lambda_{k}^{i} r_{t}^{i}(s)}{1-r_{t}^{1}(s)}>0, \zeta_{k, t-1}=\frac{\sum_{i=2}^{I} \lambda_{k}^{i} r_{t-1}^{i}}{1-r_{t-1}^{1}}>0 \\
r_{t}^{i}(s) & =\sum_{k=1}^{K} R_{k}(s) \frac{\lambda_{k}^{i} r_{t-1}^{i}}{\sum_{m=1}^{I} \lambda_{k}^{m} r_{t-1}^{m}}, r_{t-1}^{i}=r_{t-1}^{i}\left(s^{t-1}\right) .
\end{aligned}
$$

In the above formulas, we assume that $s^{t-1}$ is fixed and denote, for shortness, the value of $r_{t-1}^{i}\left(s^{t-1}\right)$ at the point $s^{t-1}$ by $r_{t-1}^{i}$. Observe that $r_{t}^{i}(s)$ coincides with $r_{t}^{i}\left(s^{t-1}, s\right)=r_{t}^{i}\left(s_{1}, \ldots, s_{t-1}, s\right)$. If $t=1$, then $r_{t-1}^{i}\left(s^{t-1}\right):=r_{0}^{i}$.

4th step. We will employ the following lemma (its proof is given in the Appendix). 
Lemma 1 For any $\mu=\left(\mu_{k}\right) \in \Delta^{K}$ with $\mu>0$ and any $\kappa \in[0,1]$, we have

$$
E \ln \sum_{k=1}^{K} R_{k}(s) \frac{\lambda_{k}^{*}}{\lambda_{k}^{*} \kappa+\mu_{k}(1-\kappa)}-E \ln \sum_{k=1}^{K} R_{k}(s) \frac{\mu_{k}}{\lambda_{k}^{*} \kappa+\mu_{k}(1-\kappa)} \geq 0 .
$$

Furthermore, if $\lambda^{*} \neq \mu$, then the difference on the left-hand side of (8) is strictly positive.

Here, $E g(s)=\sum_{s} p_{s} g(s)$ for any function $g$ on $S$. We write $\mu>0$ if all the coordinates of the vector $\mu$ are strictly positive. Recall that we have assumed $\lambda^{*}>0$.

Let us prove that the expression in (5) is bounded away from 0 by a strictly positive constant. Let us regard this expression as a function $\Phi(r)$ of the vector $r=r_{t-1}$, where $r$ ranges over $\Delta_{+}^{I}:=\left\{r \in \Delta^{I}: r>0\right\}$ (the numbers $\zeta_{k, t-1}, \zeta_{k, t}(s)$ and $r_{t}^{i}(s)$ are expressed through $r^{i}=r_{t-1}^{i}$ by formulas (6) and (7)). By virtue of Lemma $1, \Phi(r) \geq 0, r \in \Delta^{I}$. It suffices to show that $\inf _{\Delta_{+}^{I}} \Phi(r)>0$.

Let us perform the following change of variables

$$
\theta=1-r^{1}, \text { and } \gamma^{m}=\frac{r^{m}}{1-r^{1}} \text { for } m=2, \ldots, I .
$$

Thus $r^{1}=1-\theta$ and $r^{m}=\theta \gamma^{m}$. When $r$ ranges over $\Delta_{+}^{I}$, the vector $\left(\theta, \gamma^{2}, \ldots, \gamma^{I}\right)$ runs through the set $(0,1) \times \Delta_{+}^{I-1}$. Writing formulas $(6)$ and (7) in terms of the new variables, we obtain

$$
\begin{aligned}
\zeta_{k, t-1} & =\frac{\sum_{i=2}^{I} \lambda_{k}^{i} \theta \gamma^{i}}{\theta}=\sum_{i=2}^{I} \lambda_{k}^{i} \gamma^{i}, \\
r_{t}^{1}(s) & =\sum_{j=1}^{K} R_{j}(s) \frac{\lambda_{j}^{1}(1-\theta)}{\lambda_{j}^{1}(1-\theta)+\theta \zeta_{j, t-1}}, \\
r_{t}^{i}(s) & =\sum_{j=1}^{K} R_{j}(s) \frac{\lambda_{j}^{i} \theta \gamma^{i}}{\lambda_{j}^{1}(1-\theta)+\theta \zeta_{j, t-1}}, \quad i=2, \ldots, I \\
\zeta_{k, t}(s) & =\frac{\sum_{i=2}^{I} \lambda_{k}^{i} r_{t}^{i}(s)}{1-r_{t}^{1}(s)}=\frac{\sum_{j=1}^{K} R_{j}(s) \frac{\sum_{i=2}^{I} \lambda_{k}^{i} \lambda_{j}^{i} \gamma^{i}}{\lambda_{j}^{1}(1-\theta)+\theta \zeta_{j, t-1}}}{\sum_{j=1}^{K} R_{j}(s) \frac{\zeta_{j, t-1}}{\lambda_{j}^{1}(1-\theta)+\theta \zeta_{j, t-1}}}
\end{aligned}
$$


Let us consider the function $\Psi(\theta, \gamma)$ of $\theta \in[0,1]$ and $\gamma=\left(\gamma^{2}, \ldots, \gamma^{I}\right) \in$ $\Delta^{I-1}$ defined by the formula

$$
\sum_{\sigma, s} p_{\sigma} p_{s} \ln \frac{\sum_{k} \frac{R_{k}(\sigma) \lambda_{k}^{1}}{\lambda_{k}^{1} r_{t}^{1}(s)+\zeta_{k, t}(s)\left(1-r_{t}^{1}(s)\right)}}{\sum_{k} \frac{R_{k}(\sigma) \zeta_{k, t}(s)}{\lambda_{k}^{1} r_{t}^{1}(s)+\zeta_{k, t}(s)\left(1-r_{t}^{1}(s)\right)}}+\sum_{s} p_{s} \ln \frac{\sum_{k} \frac{R_{k}(s) \lambda_{k}^{1}}{\lambda_{k}^{1}(1-\theta)+\zeta_{k, t-1} \theta}}{\sum_{k} \frac{R_{k}(s) \zeta_{k, t-1}}{\lambda_{k}^{1}(1-\theta)+\zeta_{k, t-1} \theta}}
$$

where $\zeta_{k, t-1}, r_{t}^{i}(s)$, and $\zeta_{k, t}(s)$ are given by equations (10)-(13). For every $r \in \Delta_{+}^{I}$, there is an element $(\theta, \gamma) \in(0,1) \times \Delta_{+}^{I-1} \subset[0,1] \times \Delta^{I-1}$, as defined by (9), such that $\Phi(r)=\Psi(\theta, \gamma)$, and so

$$
\inf _{r \in \Delta_{+}^{I}} \Phi(r) \geq \inf _{(\theta, \gamma) \in[0,1] \times \Delta^{I-1}} \Psi(\theta, \gamma) .
$$

Thus it suffices to show that the last infimum is strictly positive.

5th step. The values of the functions $\zeta_{k, t-1}=\zeta_{k, t-1}(\gamma)$ and $\zeta_{k, t}(s)=$ $\zeta_{k, t}(s, \theta, \gamma)$ are not less than $\left(\min \lambda_{j}^{i}\right)^{3}>0$ (see (10) and (13)). Consequently, the function $\Psi(\theta, \gamma)$ is continuous on the compact set $[0,1] \times \Delta^{I-1}$, and in order to show that it is bounded away from 0 it suffices to show that it is strictly positive. Suppose $\Psi(\theta, \gamma)$ is zero for some $(\theta, \gamma)$. Then

$$
\sum_{s} p_{s} \ln \frac{\sum_{k} R_{k}(s) \lambda_{k}^{1}\left(\lambda_{k}^{1}(1-\theta)+\zeta_{k, t-1} \theta\right)^{-1}}{\sum_{k} R_{k}(s) \zeta_{k, t-1}\left(\lambda_{k}^{1}(1-\theta)+\zeta_{k, t-1} \theta\right)^{-1}}=0
$$

and

$$
\sum_{\sigma} p_{\sigma} \ln \frac{\sum_{k} R_{k}(\sigma) \lambda_{k}^{1}\left(\lambda_{k}^{1} r_{t}^{1}(s)+\zeta_{k, t}(s)\left(1-r_{t}^{1}(s)\right)\right)^{-1}}{\sum_{k} R_{k}(\sigma) \zeta_{k, t}(s)\left(\lambda_{k}^{1} r_{t}^{1}(s)+\zeta_{k, t}(s)\left(1-r_{t}^{1}(s)\right)\right)^{-1}}=0,
$$

for all $s \in S$, since, by virtue of Lemma 1, the above sums are non-negative. The former of these two equations implies

$$
\lambda_{k}^{1}=\zeta_{k, t-1}, \text { for all } k=1, \ldots, K
$$

and the latter gives

$$
\lambda_{k}^{1}=\zeta_{k, t}(s)=\sum_{j=1}^{K} \frac{R_{j}(s) \sum_{i=2}^{I} \lambda_{k}^{i} \lambda_{j}^{i} \gamma^{i}}{\lambda_{j}^{1}(1-\theta)+\zeta_{j, t-1} \theta}=\sum_{j=1}^{K}\left(\frac{R_{j}(s)}{\lambda_{j}^{*}} \sum_{i=2}^{I} \lambda_{k}^{i} \lambda_{j}^{i} \gamma^{i}\right)
$$


for all $s \in S$ and $k=1, \ldots, K$. Indeed, if (16) fails to hold, then, in view of Lemma 1, we have a strict inequality in (14). Analogously, (15) implies (17).

6th step. Let us show that relations (16) and (17) cannot hold simultaneously. This will lead to a contradiction and thus prove the theorem. Since $\lambda_{k}^{1}=\lambda_{k}^{*}$, we obtain from (10) and (16)

$$
\lambda_{k}^{*}=\zeta_{k, t-1}=\sum_{m=2}^{I} \lambda_{k}^{m} \gamma^{m}, k=1, \ldots, K
$$

By using this formula and (17), we find

$$
\sum_{j=1}^{K} R_{j}(s) \pi_{j}^{k}=0, s \in S, k=1, \ldots, K,
$$

where

$$
\pi_{j}^{k}=\frac{1}{\lambda_{j}^{*}} \sum_{m=2}^{I} \lambda_{k}^{m} \lambda_{j}^{m} \gamma^{m}-\sum_{m=2}^{I} \lambda_{k}^{m} \gamma^{m}
$$

Since there are no redundant assets, we have $\pi_{j}^{k}=0$ for each $j$ and $k$. This yields

$$
\sum_{m=2}^{I} \lambda_{k}^{m}\left(\lambda_{j}^{m}-\lambda_{j}^{*}\right) \gamma^{m}=0, \text { for all } k, j=1, \ldots, K .
$$

By adding up these equalities over $k$ and setting $k=j$ in the foregoing formula, we find

$$
\sum_{m=2}^{I}\left(\lambda_{j}^{m}-\lambda_{j}^{*}\right) \gamma^{m}=0, \text { and } \sum_{m=2}^{I} \lambda_{j}^{m}\left(\lambda_{j}^{m}-\lambda_{j}^{*}\right) \gamma^{m}=0, j=1, \ldots, K .
$$

By combining these equations, we get

$$
\sum_{m=2}^{I}\left(\lambda_{j}^{m}-\lambda_{j}^{*}\right)^{2} \gamma^{m}=0, j=1, \ldots, K
$$

Since $\sum \gamma^{m}=1$, we have $\gamma^{m}>0$ for some $m \in\{2, \ldots, I\}$. Then $\lambda_{j}^{m}=\lambda_{j}^{*}$, $j=1, \ldots, K$. This means that some investor $m \in\{2, \ldots, I\}$ uses the strategy $\left(\lambda_{1}^{*}, \ldots, \lambda_{K}^{*}\right)$. We have obtained a contradiction. 


\section{Appendix}

Proof of Lemma 1. Clearly, if $\mu=\lambda^{*}$, inequality (8) turns into an equality. Let us show that the expression on the left-hand side of $(8)$ - denote it by $\Xi(\mu, \kappa)$ - is strictly positive for all $\kappa \in[0,1]$ and $\mu \neq \lambda^{*}$. By applying Jensen's inequality, we find

$$
\begin{aligned}
& E \ln \sum_{k=1}^{K} R_{k}(s) \frac{\lambda_{k}^{*}}{\lambda_{k}^{*} \kappa+(1-\kappa) \mu_{k}} \geq \sum_{k=1}^{K} \lambda_{k}^{*} \ln \frac{\lambda_{k}^{*}}{\lambda_{k}^{*} \kappa+(1-\kappa) \mu_{k}}, \\
& E \ln \sum_{k=1}^{K} R_{k}(s) \frac{\mu_{k}}{\lambda_{k}^{*} \kappa+(1-\kappa) \mu_{k}} \leq \ln E \sum_{k=1}^{K} R_{k}(s) \frac{\mu_{k}}{\lambda_{k}^{*} \kappa+(1-\kappa) \mu_{k}}
\end{aligned}
$$

and so

$$
\Xi(\kappa, \mu) \geq \sum_{k=1}^{K} a_{k} \ln \frac{a_{k}}{a_{k} \kappa+(1-\kappa) \mu_{k}}-\ln \sum_{k=1}^{K} a_{k} \frac{\mu_{k}}{a_{k} \kappa+(1-\kappa) \mu_{k}}
$$

where $a_{k}=\lambda_{k}^{*}$.

Let $\kappa=0$. Then the right-hand side of $(21)$ reduces to

$$
\sum a_{k} \ln a_{k}-\sum a_{k} \ln \mu_{k} .
$$

This difference is strictly positive, since $\left(a_{k}\right) \neq\left(\mu_{k}\right)$.

If $\kappa \in(0,1]$, then we have a strict inequality in $(20)$. To prove this it suffices to show that the function

$$
\phi(s)=\sum_{k=1}^{K} R_{k}(s) \mu_{k}\left[\lambda_{k}^{*} \kappa+(1-\kappa) \mu_{k}\right]^{-1}, s \in S,
$$

is not a constant. Suppose $\phi(s)$ is constant, i.e. $\phi(s) \equiv \gamma$. Then

$$
\sum_{k=1}^{K} R_{k}(s)\left(\mu_{k}\left[\lambda_{k}^{*} \kappa+(1-\kappa) \mu_{k}\right]^{-1}-\gamma\right)=0, s \in S
$$

which implies $\mu_{k}=\gamma\left(\lambda_{k}^{*} \kappa+(1-\kappa) \mu_{k}\right)$, since the functions $R_{k}(\cdot), k=$ $1,2, \ldots, K$, are linearly independent. We can see that $\gamma=1$, and so $\kappa\left(\lambda_{k}^{*}-\right.$ $\left.\mu_{k}\right)=0$. Since $\kappa>0$, this implies $\lambda_{k}^{*}=\mu_{k}, k=1,2, \ldots, K$, which, however, is ruled out by our assumptions. 
It remains to prove that the expression on the right-hand side of $(21)$ is non-negative. It is equal to zero if $\kappa=1$. If $\kappa<1$, we can write it in the form

$$
g(u)=\sum_{k=1}^{K} a_{k} \ln \frac{a_{k}}{a_{k} u+\mu_{k}}-\ln \sum_{k=1}^{K} a_{k} \frac{\mu_{k}}{a_{k} u+\mu_{k}},
$$

where $u=\kappa(1-\kappa)^{-1}$. We can see that $g(u) \rightarrow 0$ as $u \rightarrow \infty$. Thus it remains to prove the inequality $g^{\prime}(u) \leq 0$ for all $u>0$. We write

$$
g^{\prime}(u)=-\sum_{k=1}^{K} a_{k}^{2}\left(a_{k} u+\mu_{k}\right)^{-1}+\frac{\sum_{k=1}^{K} a_{k}^{2} \mu_{k}\left(a_{k} u+\mu_{k}\right)^{-2}}{\sum_{k=1}^{K} a_{k} \mu_{k}\left(a_{k} u+\mu_{k}\right)^{-1}} .
$$

The sign of $g^{\prime}(u)$ is the same as the sign of the expression

$$
J:=-\left[\sum_{k=1}^{K} a_{k}^{2}\left(a_{k} u+\mu_{k}\right)^{-1}\right] \sum_{k=1}^{K} a_{k} \mu_{k}\left(a_{k} u+\mu_{k}\right)^{-1}+\sum_{k=1}^{K} a_{k}^{2} \mu_{k}\left(a_{k} u+\mu_{k}\right)^{-2} .
$$

By setting $w_{k}=a_{k} u+\mu_{k}$, we find $\mu_{k}=w_{k}-a_{k} u$ and

$$
\begin{aligned}
J & =-\left[\sum_{k=1}^{K} a_{k}^{2} w_{k}^{-1}\right] \sum_{k=1}^{K} a_{k}\left(w_{k}-a_{k} u\right) w_{k}^{-1}+\sum_{k=1}^{K} a_{k}^{2}\left(w_{k}-a_{k} u\right) w_{k}^{-2} \\
& =-\left[\sum_{k=1}^{K} a_{k}^{2} w_{k}^{-1}\right]\left[1-\sum_{k=1}^{K} a_{k}^{2} u w_{k}^{-1}\right]+\sum_{k=1}^{K} a_{k}^{2} w_{k}^{-1}-\sum_{k=1}^{K} a_{k}^{3} u w_{k}^{-2} \\
& =u\left[\left(\sum_{k=1}^{K} a_{k} v_{k}\right)^{2}-\sum_{k=1}^{K} a_{k} v_{k}^{2}\right]
\end{aligned}
$$

where $v_{k}=a_{k} w_{k}^{-1}$. The last expression is non-positive by virtue of the Schwartz inequality.

The lemma is proved.

\section{References}

Algoet, P. H., And T. M. Cover (1988): "Asymptotic Optimality and Asymptotic Equipartition Properties of Log-Optimum Investment," $A n$ nals of Probability, 16, 876-898. 
Aurell, E., R. Baviera, O. Hammarlid, M. Serva, and A. Vulpiani (1998): "Gambling and Pricing of Derivatives," Working Paper, SSRN Electronic Paper Collection (http://papers.ssrn.com/).

Blume, L., And D. Easley (1992): "Evolution and Market Behavior," Journal of Economic Theory, 58, 9-40.

(2000): "If You're So Smart, Why Aren't You Rich? Belief Selection in Complete and Incomplete Markets," mimeo, Department of Economics, Cornell University.

Breiman, L. (1961): "Optimal gambling systems for favorable games," Fourth Berkely Symposium on Math. Statist. and Probability, 1, 65-78.

Browne, S., And W. Whitt (1996): "Portfolio Choice and the Bayesian Kelly Criterion," Advances in Applied Probability, 28, 1145-1176.

Cover, T. (1991): "Universal Portfolios," Mathematical Finance, 1, 1-29.

Hakansson, N. H. (1970): "Optimal investment and consumption strategies under risk for a class of utility functions," Econometrica, 38, 587-607.

Hakansson, N. H., and W. T. Ziemba (1995): "Capital Growth Theory," in Handbooks in Operations Research and Management Science, Volume 9, Finance, ed. by R. A. Jarrow, V. Maksimovic, and W. T. Ziemba, chap. 3, pp. 65-86. Elsevier, Amsterdam.

Hall, P., And C. Heyde (1980): Martingale limit theory and its application. Academic Press, London.

Hens, T., and K. R. Schenk-Hoppé (2001): "An Evolutionary Portfolio Theory," Working Paper No. 74, Institute for Empirical Research in Economics, University of Zurich.

Karatzas, I., And S. Shreve (1998): Methods of Mathematical Finance. Springer-Verlag, New York.

Kelly, J. (1956): "A New Interpretation of Information Rate," Bell System Technical Journal, 35, 917-926.

Sandroni, A. (2000): "Do Markets Favor Agents Able to Make Accurate Predictions?," Econometrica, 68, 1303-1341. 
Thorp, E. (1971): "Portfolio choice and the Kelly criterion," In Stochastic Models in Finance,W.T. Ziemba and R.G. Vickson, eds., 599-619. 